\title{
Comprendre les objets connectés grand public : proposition d'une taxonomie centrée sur l'utilisateur
}

\author{
`Zeling ZHONG \\ Ingénieure de recherche \\ Université Paris-Saclay, Univ Evry, IMT-BS, LITEM, 91025, Evry-Courcouronnes, France \\ Christine BALAGUE \\ Professeure en Sciences de Gestion \\ Université Paris-Saclay, Univ Evry, IMT-BS, LITEM, 91025, Evry-Courcouronnes, France
}

\section{Résumé :}

Depuis ces dernières années, les objets connectés suscitent un intérêt croissant dans le cadre de la consumérisation des TIC, influençant notre vie quotidienne. Néanmoins, la numérisation des objets physiques offre une abondance d'objets apparemment similaires mais caractérisés par certains attributs que les consommateurs ne parviennent pas à s'apercevoir et à s'approprier. En raison du phénomène de désengagement, les consommateurs ont besoin d'un outil pour les guider dans le choix de l'objet connecté le plus appropriable pour répondre à leurs besoins. Cette recherche vise à mieux comprendre les objets connectés en proposant une taxonomie en fonction des niveaux d'amélioration de l'expérience et de la pertinence pour des usages effectifs tels que appropriés. Cette taxonomie centrée sur l'utilisateur nous a permis de formuler des recommandations aux acteurs du marché des objets connectés à destination du grand public, spécifique à chaque catégorie d'objets connectés.

Mots clés : objet connecté, taxonomie, usages effectifs

Abstract: In recent years, there has been an increasing interest in smart connected objects as part of the widespread IT consumerization, influencing our daily lives. Nevertheless, digitizing physical things offers an abundance of objects apparently similar but characterized by some features that the average consumers fail to perceive and to appropriate. Due to the phenomenon of users' disengagement with loT products, consumers need a tool to guide them in choosing the most suitable smart connected object to satisfy their purposes. The current research aims to understand better smart connected objects by proposing a taxonomy based on levels of providing experience enhancements and relevance for effective uses as appropriated by consumers. The user-centered taxonomy allowed us to formulate recommendations for managers and practitioners in the market for smart connected objects aimed at the public, specific to each category of smart connected objects. 
Keywords: Smart connected object, taxonomy, effective uses

\section{Introduction}

A l'heure actuelle, une révolution numérique vers «tout connecté » est en marche. Des milliards d'objets physiques de notre vie quotidienne deviendront connectables grâce à l'internet des objets. Ce dernier permet de faire dialoguer les objets du quotidien entre eux-mêmes ainsi qu'avec les êtres humains en temps réel. L'internet des objets (raccourci en l'acronyme anglais "IoT" pour " Internet of Things ") peut s'appliquer à de nombreux domaines. Par exemple, les dispositifs connectés de santé permettent de surveiller l'état de santé des patients 24 heures sur 24, et d'établir automatiquement des diagnostics très précis sur la base de données collectées; dans l'agriculture, les régulateurs connectés permettent d'utiliser des ressources de manière plus écologique en les ajustant en temps réel en fonction des besoins en engrais et en eau détectés dans l'environnement ; les montres et bracelets connectés permettent de surveiller l'état physique de porteur et de l'alerter des entraînements sportifs sur la base de données corporelles. Dans les usines, I'loT permet de garantir une production plus efficace grâce à l'interconnexion des machines en améliorant la traçabilité des produits. D'après une étude réalisée par loT Analytics (2018), 21,5 milliards d'objets connectés seraient en circulation à travers le monde entier d'ici 2025 (ce chiffre n'inclut pas les smartphones, les tablettes, les ordinateurs ou les téléphones fixes. Si inclus, il y aura 34,2 milliards d'appareils actifs mis en réseau via l'loT), les dépenses mondiales dans le secteur de l'loT s'élèveraient à 1567 milliards de dollars d'ici 2025.

Malgré annoncé comme en plein essor dans sa globalité, le marché des objets connectés a connu un retard au démarrage dans le domaine du grand public (GFK, 2014), si nous comparons avec les champs d'application multiples et fulgurants dans l'industrie. La propension des consommateurs à s'approprier les objets connectés semblerait relativement faible. La durée de l'utilisation régulière des objets connectés ne dépasse pas six mois pour un tiers d'utilisateurs. L'attitude des consommateurs à l'égard de ces objets en émergence est ambivalente (Ardelet et al., 2017). Cette attitude ambivalente peut parfois susciter une résistance aux objets connectés de la part des consommateurs (Chouk \& Mani, 2016). Le succès des produits technologiques innovants de consommation sur le marché est largement conditionné par la manière dont les utilisateurs perçoivent, utilisent et intègrent le nouvel objet technologique de consommation dans leurs pratiques quotidiennes (Orlikowski, 1992; Rogers, 1983), d'où vient la nécessité de mener des réflexions sur l'appropriabilité des objets connectés par ses utilisateurs potentiels. Les objets connectés à destination du grand public n'ont de valeur que s'ils sont intégrés dans la vie quotidienne en produisant activement des données d'usage qui les rendront intelligents, grâce à l'exploitation et à l'analyse de ces données permettant d'aider les utilisateurs dans leurs prises de décision. Par ailleurs, les objets connectés transforment radicalement le rapport de l'individu à l'objet technologique de consommation, orientant vers un rapport homme-machine indissociable qui permet à l'individu d'interagir davantage en direct sur son propre quotidien. En raison des phénomènes de désengagement vis-à-vis des objets connectés, les consommateurs ont besoin d'un outil pour les guider dans le choix 
de l'objet connecté le plus appropriable pour mieux répondre à leurs besoins et attentes. Les usages effectifs d'un même objet connecté peuvent varier d'un individu à l'autre, cette différence en termes de modes d'usages effectifs pourra nous servir d'un critère important pour proposer une nouvelle taxonomie des objets connectés en prisant en compte le rôle crucial d'utilisateurs.

Afin d'atteindre les objectifs de recherche susmentionnés, tout d'abord, nous procédons à une revue de littérature sur les définitions, les caractéristiques et les typologies existantes d'objets connectés. Ensuite, nous procédons à une étude qualitative exploratoire en nous basant sur les entretiens semi-directifs. L'ensemble des résultats de cette recherche nous a permis d'identifier les différents modes d'usages effectifs dans le processus d'appropriation des objets connectés et de proposer une taxonomie des objets connectés en fonction des niveaux d'amélioration de l'expérience et de la pertinence pour des usages effectifs tels que appropriés par les consommateurs. Enfin, nous concluons en évoquant les implications de recherche ainsi que les voies futures de recherche.

\section{Revue de littérature}

\section{1 objet connecté : un concept en constante évolution}

Le premier objet connecté a été créé en 1990 par l'Université de Carnegie Mellon. II s'agit d'un distributeur de boissons avec des capteurs embarqués qui sont reliés à un serveur du département de l'informatique. Ce distributeur connecté était capable de communiquer en temps réel son état concernant le nombre de boissons et l'heure d'arrivée de chaque boisson dans le distributeur (The Carnegie Mellon University Coke Machine, 1990), cela a permis aux étudiants de ne plus prendre des boissons encore tièdes ou de se trouver devant un distributeur vide. L'objet connecté est une expression initialement inventée par les chercheurs en informatique, l'idée est qu'un certain niveau d'intelligence est intégré dans les objets du quotidien, ce qui les rend communicant et mettant en réseau.

A ce jour, le terme d'objet connecté ne possède pas encore de définition normative et officielle, qui peut s'expliquer par le fait que l'expression de cet objet en émergence est encore jeune ainsi que les technologies de l'information et de la communication liées à cet objet sont en constante évolution. Depuis ces dernières décennies, les objets connectés suscitent un intérêt de recherche considérable en sciences humaines et sociales. Diverses disciplines ont apporté leurs propres définitions et appellations, cela rend difficile un consensus sur la définition de cet objet en émergence dans la littérature existante. Sur la base de notre revue de littérature, une convergence entre la dimension " connectivité " et la dimension " objet » se trouve dans de nombreuses définitions existantes d'objets connectés. Toutefois, chaque dimension constructive qui semble intuitive n'a pas été définie de manière cohérente dans les travaux existants. En ce qui concerne l'aspect " objet ", la littérature n'a pas abouti à un consensus sur le rôle des dispositifs mobiles (les PCs, les tablettes et les smartphones) à l'ère des objets connectés. Ils sont exclus dans des définitions (ex: McKinsey Global Institute, 2015; Oberländer et al., 2018; Püschel et al., 2016), mais inclus dans d'autres (ex: Atzori, lera \& 
Morabito, 2014; Benghozi, Bureau \& Massit-Folléa, 2015; Sundmaeker et al., 2010). En ce qui concerne l'aspect " connectivité ", l'objet connecté a été défini de manière hétérogène dans la littérature, qui s'explique par le fait du manque d'un standard de communication pour tous les objets connectés à ce jour (Benghozi, Bureau \& Massit-Folléa, 2015).

Afin de mieux répondre à notre questionnement dans le cadre de cette recherche, nous proposons une définition adaptée: « un objet connecté est un objet physique équipé de capteurs et de processeurs auquel l'ajout d'une connexion réseau lui permet de proposer de nouveaux services pour transcender son usage initial " (Zhong, 2019). Concernant la dimension « objet », nous sommes en ligne avec le premier groupe de définitions. Les dispositifs mobiles (tablettes et smartphones et PCs) avec accès à internet dont la première vocation est de recevoir les informations intentionnelles saisies par les êtres humains, qui ne servent que de passerelle transitoire vers un réseau pour certains objets connectés non autonomes, ont été exclus. Concernant la dimension «connectivité », nous sommes en ligne avec les définitions existantes, compte tenu de l'inexistence d'une technologie de communication parfaite pour tous les cas d'usage à ce jour. Plus précisément, il s'agit des objets physiques d'usages familiers qui existent initialement indépendamment des TICs, qui deviennent capables de recevoir et d'envoyer des informations via une connexion directe ou indirecte à un réseau de communication (ex : des réseaux radio bas-débit à longue portée de type LoRa ou Sigfox, ZigBee, réseaux cellulaires mobiles, NFC, WIFI, LIFI, Bluetooth). Une dimension supplémentaire de la connexion réseau permet à ces objets d'usages familiers de fournir de nouveaux services en proposant des "fonctions augmentées " par rapport à leurs usages initiaux. Prenons l'exemple d'une montre connectée, l'ajout d'une connexion réseau lui apporte une valeur supplémentaire par rapport à une montre traditionnelle, lorsqu'elle est connectée à une plateforme de santé, qui va largement au-delà de son usage initial de donner l'heure.

\subsection{Caractérisation d'un objet connecté}

Dans le cadre de cette recherche, nous nous intéressons à l'appropriabilité des objets connectés grand public par les utilisateurs potentiels. Afin de mieux comprendre notre objet de recherche, cette sous-section est destinée à caractériser les objets connectés. Sur la base d'une revue de littérature lié à ce sujet, l'objet connecté possède généralement les six caractéristiques synthétisées dans le tableau 1.

\begin{tabular}{|c|c|c|}
\hline Caractéristiques & & Références \\
\hline Autogestion & $\begin{array}{l}\text { Cela fait référence à la capacité } \\
\text { d'apprendre à partir des } \\
\text { expériences antérieures pour } \\
\text { s'adapter aux situations et de } \\
\text { gérer son cycle de vie pour } \\
\text { s'optimiser continuellement en se } \\
\text { basant sur l'ensemble des } \\
\text { données récoltées et analysées. }\end{array}$ & $\begin{array}{l}\text { Meyer, Främling \& Holmström } \\
\text { (2009); López et al. (2011), } \\
\text { Smutný (2016); Hernández \& } \\
\text { Reiff-Marganiec (2014); } \\
\text { Kiritsis (2011); Ning \& Hu } \\
\text { (2012), Kawsar et al. (2010) }\end{array}$ \\
\hline $\begin{array}{l}\text { Traitement } \& \\
\text { programmation }\end{array}$ & $\begin{array}{l}\text { Le traitement fait référence à la } \\
\text { capacité d'exécuter des }\end{array}$ & $\begin{array}{l}\text { Meyer, Främling \& Holmström } \\
\text { (2009); Atzori, lera \& Morabito }\end{array}$ \\
\hline
\end{tabular}




\begin{tabular}{|c|c|c|}
\hline & 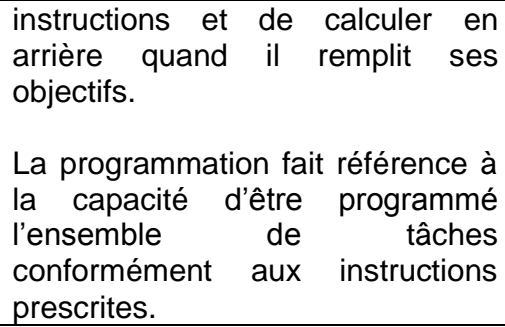 & $\begin{array}{l}\text { (2014); López et al. (2011); } \\
\text { Hernández \& Reiff-Marganiec } \\
\text { (2014); Yoo (2010), Smutný } \\
\text { (2016); Kawsar et al. (2010), } \\
\text { Püschel et al. (2016); Kiritsis } \\
\text { (2011); Ning \& Hu (2012); } \\
\text { Fortino, Rovella, Russo \& } \\
\text { Savaglio (2014) }\end{array}$ \\
\hline $\begin{array}{ll}\text { Détection } & \& \\
\text { actionnement } & \end{array}$ & $\begin{array}{l}\text { La détection fait référence à la } \\
\text { capacité de récolter des données } \\
\text { à partir de sa propre structure ou } \\
\text { de son environnement (ex : la } \\
\text { maison, le corps humain). } \\
\text { L'actionnement fait référence à la } \\
\text { capacité de provoquer un } \\
\text { changement sur sa propre } \\
\text { structure ou sur son } \\
\text { environnement. } \\
\text { Grâce à ces deux capacités, l'objet } \\
\text { connecté est conscient de son } \\
\text { entourage et de son propre état. II } \\
\text { est en mesure d'agir et de réagir } \\
\text { pour accomplir des tâches et } \\
\text { d'améliorer son propre état. }\end{array}$ & $\begin{array}{l}\text { Kortuem et al. (2010); Meyer, } \\
\text { Främling \& Holmström } \\
(2009) ; \quad \text { Smutný (2016); } \\
\text { Kiritsis (2011) } \\
\text { Uckelmann, Harrison \& } \\
\text { Michahelles (2011); Kawsar, } \\
\text { Nakajima, Park \& Yeo (2010); } \\
\text { Atzori, lera \& Morabito (2014); } \\
\text { Fortino, Rovella, Russo \& } \\
\text { Savaglio (2014); López et al. } \\
\text { (2011); Hernández \& Reiff- } \\
\text { Marganiec (2014); Porter \& } \\
\text { Heppelmann (2014); Püschel } \\
\text { et al. (2016) }\end{array}$ \\
\hline Réseautage & $\begin{array}{l}\text { Cela fait référence à la capacité } \\
\text { d'échanger les données dans les } \\
\text { mondes physiques et virtuels } \\
\text { grâce à la mise en réseau avec un } \\
\text { grand nombre de systèmes de } \\
\text { réseaux et d'objets permettant de } \\
\text { supporter de différents modèles de } \\
\text { communication (ex: modèle un à } \\
\text { un, modèle plusieurs à un, modèle } \\
\text { un à plusieurs, modèle plusieurs à } \\
\text { plusieurs). }\end{array}$ & $\begin{array}{l}\text { Kortuem et al. (2010) ; Meyer, } \\
\text { Främling \& Holmström } \\
(2009) ; \text { Yoo (2010); Kiritsis } \\
(2011) \text {; Moawad et al. } \\
(2012) \text {; Porter \& Heppelmann } \\
(2014) \text {; Hernández \& Reiff- } \\
\text { Marganiec (2014); Atzori, lera } \\
\text { \& Morabito (2014); López et } \\
\text { al. (2011); Ning \& Hu (2012) }\end{array}$ \\
\hline Communication & $\begin{array}{l}\text { Cela fait référence à la capacité de } \\
\text { reporter son état et de } \\
\text { communiquer son environnement } \\
\text { (ex: la température, l'humidité, le } \\
\text { niveau de vibration) avec d'autres } \\
\text { objets, systèmes ou utilisateurs. }\end{array}$ & $\begin{array}{l}\text { Meyer, Främling \& Holmström } \\
\text { (2009); Atzori, lera \& Morabito } \\
\text { (2014); Kiritsis (2011); Smutný } \\
\text { (2016); Uckelmann, Harrison } \\
\& \quad \text { Michahelles (2011); } \\
\text { Moawad et al. (2012) }\end{array}$ \\
\hline Identification & $\begin{array}{l}\text { Cela fait référence à la capacité de } \\
\text { s'identifier aux autres systèmes ou } \\
\text { objets, grâce à son identité unique } \\
\text { et immuable (ex: une adresse IP, } \\
\text { une puce RFID, un code barre). }\end{array}$ & $\begin{array}{l}\text { Meyer, Främling \& Holmström } \\
\text { (2009); López et al. (2011); } \\
\text { Kiritsis (2011); Uckelmann, } \\
\text { Harrison \& Michahelles } \\
\text { (2011); Hernández \& Reiff- } \\
\text { Marganiec (2014); Fortino, } \\
\text { Rovella, Russo \& Savaglio } \\
\text { (2014); Kawsar, Nakajima, } \\
\text { Park \& Yeo (2010); Ning \& } \\
\text { Hu (2012) }\end{array}$ \\
\hline
\end{tabular}


D'une manière générale, un objet connecté est un objet intelligent doté des capacités fonctionnelles très hétérogènes en identification, communication, réseautage, détection \& actionnement, traitement \& programmation, autogestion. Plus un objet connecté possède un nombre élevé de six capacités/ caractéristiques susmentionnées, plus il est considéré par le grand public comme " ayant un niveau élevé d'intelligence ». Un objet connecté à destination du grand public est d'abord un objet physique du quotidien qui est capable de réaliser une certaine fonction de base (ex: une montre traditionnelle permet de donner l'heure), l'ajout d'une connexion réseau lui permet de fournir de nouveaux services pour transcender sa fonction initiale. Les caractéristiques essentielles de l'objet connecté sont que les technologies de communication associées transforment des objets ordinaires en dispositifs intelligents capables de réaliser des fonctions augmentées. Grâce à leur identité numérique, ils deviennent capable de s'identifier aux autres objets et systèmes, de reporter ses états via des capteurs et d'échanger des données avec d'autres objets ou systèmes via un Cloud. Les processeurs intégrés permettent de gérer automatiquement leur propre état et leur environnement selon les instructions précédemment programmées. Grâce à la mise réseau avec des systèmes analytiques du Machine Learning et de l'Intelligence Artificielle, ils deviennent également capables de tirer des conclusions à partir d'expériences antérieures et de généraliser des actions face aux changements situationnels pour s'optimiser progressivement.

\subsection{Taxonomie des objets connectés}

Dans la littérature académique, trois grandes catégories de taxonomies des objets connectés peuvent être distinguées :

Taxonomie centrée sur la technologie : la première catégorie de taxonomie des objets connectés s'intéresse au produit vu comme une "entité technologique " et peut se scinder en deux classes principales de taxonomies: En dehors des taxonomies monocritères qui distinguent les objets connectés en fonction d'un seul critère technique tel que le niveau d'intelligence (Kiritsis, 2011), la capacité d'interaction sociale (Atzori, lera \& Morabito, 2014; Moawad et al., 2012), un grand nombre de chercheurs en provenance de diverses disciplines proposent des taxonomies centrées sur la technologie en prisant en compte plusieurs critères techniques (Ex: Fortino et al., 2014; Kawsar et al., 2010; Kortuem et al., 2010; López et al., 2011; Meyer, Främling \& Holmström, 2009; Ning \& Hu, 2012; Porter \& Heppelmann, 2014; Püschel et al., 2016; Yoo et al., 2010). L'idée de ces taxonomies multicritères est de considérer les objets connectés comme un tas technologique multicouche. Par exemple, la taxonomie de Püschel et al. (2016) comporte quatre couches, à savoir objet, interaction, données et service, ce qui est comparable aux quatre couches proposées par Yoo et al. (2010) soit service, contenu, réseau et dispositif.

- Taxonomie centrée sur l'utilisateur : la deuxième catégorie de taxonomies des objets connectés s'intéresse à la relation entre le produit et l'utilisateur. 
Smutný (2016) a proposé une taxonomie selon la relation de l'objet connecté avec l'utilisateur au centre: Surroudables (les objets connectés autour de l'utilisateur), Holdables (les objets connectés près de l'utilisateur), Wearables (les objets connectés sur l'utilisateur), et Embeddables (les objets connectés dans l'utilisateur). La taxonomie de Smutný (2016) nous paraît très intéressante vu que le rôle central de l'utilisateur a été souligné, ce qui n'a pas été pris en compte par la majorité des taxonomies existantes dans la littérature. Toutefois, les travaux de Smutný (2016) n'ont qu'étudié le rôle de l'utilisateur de manière très simple dans la taxonomie proposée.

- Taxonomie neutre : cette catégorie de taxonomies neutres des objets connectés ne s'intéresse ni au produit vu comme une entité technologique, ni à la relation entre le produit et l'être humain. Les travaux d'Uckelmann, Harrison \& Michahelles (2011) ont rentré dans la troisième catégorie, ces auteurs ont proposé une taxonomie en fonction de la finalité et du créateur Ready-made si les objets connectés sont créés pour une finalité commerciale par les professionnels, et Self-made si les objets connectés sont créés pour un usage personnel par les individus. Les travaux d'Uckelmann, Harrison \& Michahelles (2011) ont apporté une vision différente si nous comparons avec les taxonomies existantes qui se focalisent principalement sur les caractéristiques techniques des objets connectés. Néanmoins, il n'y a que deux facteurs (la finalité et le créateur) qui sont pris en considération dans la taxonomie d'Uckelmann, Harrison \& Michahelles (2011).

La taxonomie centrée sur la technologie reste prédominante dans les travaux existants relatifs à la taxonomie des objets connectés. La majorité des taxonomies existantes se sont focalisées sur l'aspect technique, à ce jour, assez peu de recherches liées à ce sujet permettent de comprendre le rôle des utilisateurs dans les écosystèmes des objets connectés. Cela peut s'expliquer par le fait qu'au début de l'ère des objets connectés, les utilisateurs sont largement considérés comme des récepteurs passifs qui ne sont que bénéficiaires de ces produits innovants et des services associées. Toutefois, le rapport homme machine devient indissociable dans un monde de plus en plus connecté. Grâce à l'analyse régulière des données d'usage, les objets connectés peuvent tirer des conclusions et s'optimiser sans cesse à partir des expériences antérieures partagées par les utilisateurs. Par conséquent, la prise en compte du rôle actif des utilisateurs est indispensable pour bien appréhender ces objets technologiques en émergence. Les taxonomies centrées sur la technologie sont destinées aux fabricants qui visent à maximiser la potentialité de leurs produits et systèmes, l'appropriabilité de ces produits technologiques innovants par les consommateurs est toutefois relativement négligée.

L'objectif de notre recherche est ainsi de proposer une taxonomie des objets connectés qui pourrait servir d'un outil orienté vers l'utilisateur pour le guider dans le choix de l'objet connecté le plus appropriable, conduisant les consommateurs à l'intégration de ces produits technologiques innovants dans leurs pratiques quotidiennes. La présente recherche s'inscrit dans la deuxième perspective et 
souligne le rôle crucial de l'utilisateur dans les écosystèmes des objets connectés. Une vision appropriative sur les objets connectés à destination du grand public a été adoptée. En d'autres termes, nous considérons les utilisateurs comme à la fois bénéficiaires et coconstructeurs de leurs objets connectés dans le cadre de cette recherche.

\section{Fondements théoriques}

Les expériences antérieures d'usage des technologies sont largement considérées comme un moteur du comportement futur de la part des individus. Les travaux d'Orlikowski (2000) soutiennent que les expériences des utilisateurs ne sont pas seulement déterminées par les possibilités de la technologie ou les éléments de la machine fournis par le système, mais plutôt par l'application des utilisateurs de leurs propres "connaissances, pouvoir, compétences et hypothèses" dans leurs pratiques d'appropriation de l'ensemble de ces éléments. L'appropriation a été définie comme la manière dont les individus utilisent, adaptent et intègrent les artefacts technologiques dans leurs pratiques quotidiennes, leur travail ou leurs loisirs (Bar, Pisani \& Weber, 2016; Carroll et al., 2003). Au cours du processus d'appropriation, la participation active des utilisateurs transforme la "technologie telle que conçue" en "technologie telle qu'utilisée" (Carroll, 2004). À cet égard, les résultats du processus d'appropriation d'une technologie par les individus se réfèrent aux usages effectifs résultant de leur interaction avec la technologie et de la négociation continue des utilisateurs sur celle-ci (Zhong et al., 2017). Etant considéré comme produit technologique innovant, le cycle de vie de l'utilisation d'un objet connecté peut être expliqué par le modèle d'appropriation de la technologie de Carroll (2004). Cet auteur souligne que les consommateurs complètent la conception d'une technologie en se l'appropriant. Cela se traduit par le passage depuis "la technologie telle que conçue ", qui implique les usages prescrits associés à la technologie initialement présentée aux consommateurs, jusqu'à "la technologie telle que conçue », qui implique les usages effectifs associés à la technologie adaptée et incorporée dans la vie quotidienne des utilisateurs.

Sandström et al. (2008) soutiennent que la valeur pour l'utilisateur dérivée d'un service basé sur la technologie réside dans les expériences qui résultent de l'utilisation du service, mais pas dans le service lui-même. Les expériences de nature médiatisée par la technologie peuvent effectivement améliorer les bénéfices des utilisateurs. Neuhofer, Buhalis \& Ladkin (2014) ont développé un modèle de hiérarchie de l'expérience pour mieux comprendre l'expérience utilisateur telle que facilitée par la technologie et son potentiel de la création de valeur par le biais de la co-création d'expériences. Ces auteurs ont identifié trois niveaux d'expériences médiatisées par la technologie. Au niveau des expériences assistées par la technologie, les technologies jouent un rôle d'assistance à l'utilisateur pour accéder à des sites web non interactifs. Ces expériences sont caractérisées par les technologies du web 1.0, telles que les systèmes de réservation et de distribution proposés par les applications mobiles, dans lesquelles les utilisateurs ne peuvent pas interagir pleinement ou co-créer leurs expériences. Au niveau des expériences enrichies par la technologie, les technologies permettent aux utilisateurs de participer activement à la création de leurs expériences et de les façonner. Les 
utilisateurs peuvent interagir avec les organisations grâce aux technologies interactives disponibles sur le Web 2.0, telles que les plateformes de médias sociaux. Au niveau des expériences habilitées par la technologie, les technologies deviennent une partie indispensable de l'expérience, contrairement aux deux types précédents d'expériences dans lesquels les technologies ne jouent qu'un rôle de soutien, les technologies sont omniprésentes à ce niveau et doivent exister pour que l'expérience de l'utilisateur ait lieu. L'intégration de technologies de l'information et de la communication sophistiquées permet aux utilisateurs d'interagir pleinement et de prendre part à la co-création d'expériences. Les objets connectés grand public sont des objets physiques quotidiens auxquels l'ajout d'une connexion réseau permettent de transcender leur usage initial en proposant de nouveaux services technologiques liées à la connexion réseau. Ainsi, il nous semble pertinent d'adopter le modèle de hiérarchie de l'expérience de Neuhofer, Buhalis \& Ladkin (2014) pour comprendre les expériences des utilisateurs médiatisées par les objets connectés grand public.

\section{Méthodologie de recherche}

En vue de mieux comprendre les expériences d'usage des objets connectés et d'identifier les modes d'usages effectifs à l'issue du processus d'appropriation, dixhuit entretiens semi-directifs d'une durée moyenne de cinquante-quatre minutes ont été menées auprès d'utilisateurs français de montres connectées. La nature d'appropriation met l'accent sur les usages effectifs ou les modes d'usages appropriés par les utilisateurs (Bar, Pisani \& Weber, 2016). Les usages effectifs des objets connectés peuvent être considérés comme les produits/résultats du processus d'appropriation. Les entretiens réalisés ont été intégralement retranscrits. Un peu plus de 180 pages de discours recueillis ont fait l'objet d'une analyse de contenu thématique (voir annexes 1 et 2 pour plus de détails). Suivant les recommandations de Gavard-Perret et al. (2012), le travail d'analyse a été réalisé par deux chercheuses. Chaque chercheuse a développé de manière indépendante des catégories et examiné de manière critique l'analyse préliminaire de l'autre. Sur la base d'une grille définitive validée par le consensus de ces deux chercheuses, le travail de codage sur l'ensemble de retranscriptions a été fait par chacune de ces deux chercheuses. La valeur de l'indice de fiabilité inter-codeurs a été calculée par une troisième chercheuse, le pourcentage de l'accord sur l'analyse finale entre les deux chercheuses - codeuses s'élève à $81 \%$.

\section{Résultats}

Trois principaux résultats de cette étude sont présentés dans la figure 1 . Premièrement, sur la base de la revue de littérature relatives aux caractéristiques des objets connectés, en combinant des concepts similaires, 6 catégories de capacités fonctionnelles des objets connectés ont été dérivées: I'identification, la communication, le réseautage, la détection \& l'actionnement, le traitement \& la programmation, l'autogestion. Les expériences médiatisées par les objets connectés ont été ensuite classées en trois niveaux d'expérience utilisateur du 
moins sophistiqué au plus sophistiquée selon le degré d'amélioration des expériences médiatisées par les six catégories de capacités fonctionnelles des objets connectés : les expériences assistées par les capacités, les expériences enrichies par les capacités, et les expériences habilitées par les capacités. Deuxièmement, à partir de l'analyse thématique des discours recueillis, nous avons identifié 4 modes d'usages effectifs résultant de différents niveaux du processus d'appropriation par les utilisateurs, à savoir la routinisation des usages extra rôles, la redéfinition de l'objectif initial des concepteurs, la personnalisation, et la répétition d'usages antérieurs maitrisés. Troisièmement, nous avons établi une grille qui peut aider les consommateurs à choisir l'objet connecté le plus appropriable pour répondre à leurs besoins grâce à la taxonomie basée sur le niveau d'amélioration de l'expérience utilisateur des objets connectés et la pertinence pour des modes d'usages effectifs qui sont considérés comme les résultats de différents niveaux d'appropriation des utilisateurs: les objets connectés tels que conçus, les objets connectés tels que co-créés, et les objets connectés tels que créés par soi-même.

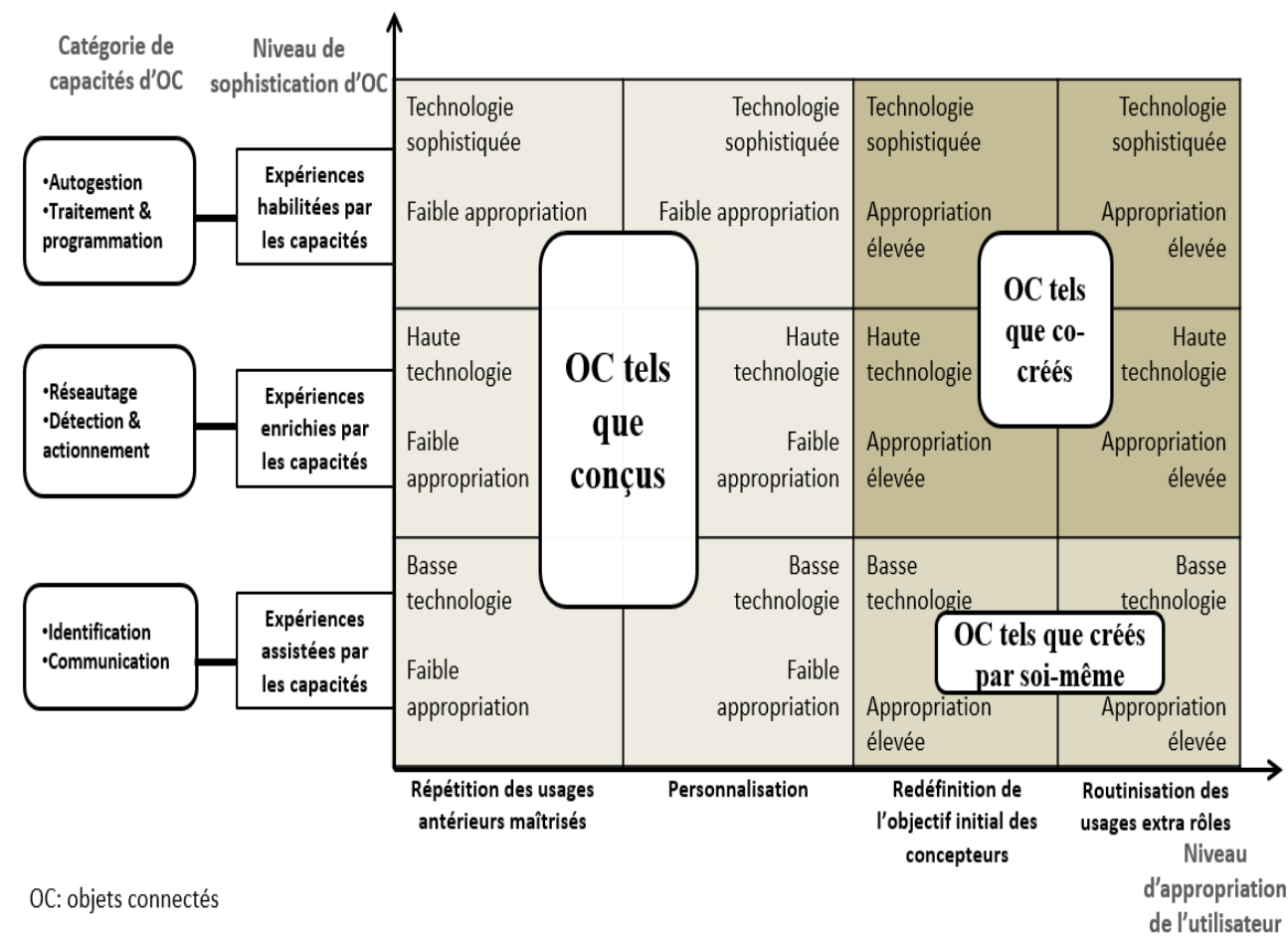

Figure 1 Taxonomie des objets connectés basée sur le niveau de sophistication des capacités et le niveau d'appropriation de l'utilisateur

\section{1- Les expériences des utilisateurs améliorées par les capacités des objets connectés}


Six catégories de capacités des objets connectés ont été dérivées de notre revue de littérature. En nous basant sur le modèle de hiérarchie de l'expérience de Neuhofer, Buhalis \& Ladkin (2014), ces capacités des objets connectés ont été classées en trois niveaux d'expérience utilisateur dans l'ordre de l'amélioration de l'expérience des objets connectés de la moins sophistiquée à la plus sophistiquée. Les trois niveaux d'expérience utilisateur médiatisées par les objets connectés peuvent être décrits comme suivante.

Niveau 1: les expériences assistées par les capacités sont des expériences utilisateurs qui incluent la moindre expérience d'interaction des objets connectés et contiennent les capacités les moins sophistiquées des objets connectés qui ne fournissent que des informations. Les capacités des objets connectés à ce niveau comportent : l'identification (il fait référence à la capacité de l'objet connecté à s'identifier aux autres objets ou systèmes, son identité unique et immuable lui permet l'accès aux informations et la présence de l'objet connecté dans un contexte numérique), la communication (il s'agit de la capacité de l'objet connecté à communiquer ses informations avec d'autres systèmes, objets ou utilisateurs). Les objets connectés les plus simples, qui sont un mécanisme de base point à point avec de nombreuses restrictions, sont inclus dans ce niveau. Par exemple, les objets physiques dotés d'une étiquette RFID.

Niveau 2: au niveau d'expériences enrichies par les capacités, les utilisateurs peuvent interagir avec l'objet connecté et tirer parti de ses capacités technologiques plus sophistiquées pour façonner leurs expériences créées. Les catégories de capacités des objets connectés à ce niveau sont : le réseautage (il s'agit d'une évolution des capacités de communication, y compris des fonctions plus complexes. II fait référence à la capacité de l'objet connecté à joindre de nombreux réseaux d'objets et de systèmes pour prendre en charge de différents modèles de communication tels que le modèle un-à-un, le modèle plusieurs-à-un, et le modèle un-à-plusieurs), la détection et l'actionnement (la détection désigne la capacité de l'objet connecté à recueillir des informations en direct à partir d'un environnement externe tel que le corps humain et la cuisine, ou à partir de sa propre structure. L'actionnement fait référence à la capacité de l'objet connecté à provoquer un changement dans l'environnement ou dans sa propre structure).

Niveau 3 : le niveau d'expérience habilitée par les capacités est considéré comme le niveau d'expérience le plus sophistiqué pour l'utilisateur. Les capacités à ce niveau fournissent une variété de solutions technologiques permettant aux utilisateurs de participer activement à la co-création d'expériences. Les capacités attribuées à ce niveau sont les suivantes : traitement et programmation (le traitement désigne la capacité de l'objet connecté à exécuter des instructions et à calculer en arrière-plan lorsqu'il atteint ses objectifs. La programmation fait référence à la capacité de l'objet connecté à être programmé pour pouvoir accomplir les différentes tâches selon les instructions initialement établies par des programmeurs), l'autogestion (il s'agit de la capacité d'utiliser les informations collectées pour gérer le cycle de vie de l'objet connecté et de la capacité d'apprendre des expériences antérieures pour s'optimiser continument son fonctionnement). 


\section{2- Les usages effectifs résultant de différents niveaux d'appropriation des objets connectés par les utilisateurs}

A partir de l'analyse des discours recueillis relatifs aux expériences réelles d'usage des montres connectées, nous avons identifié quatre modes d'usages effectifs qui résultent de niveaux croissants d'appropriation des montres connectées par les utilisateurs : la répétition d'usages antérieurs maîtrisés (les utilisateurs transfèrent leurs compétences et leurs connaissances liées à des appareils mobiles existants à l'objet connecté en répétant certains usages maîtrisés sur ces appareils mobiles qu'ils possédaient), la personnalisation (il s'agit de modifications que les concepteurs permettent à l'utilisateur de faire, effectuées par l'utilisateur afin d'individualiser son objet connecté. Chaque objet connecté devient presque unique sous ce mode d'usage effectif), la redéfinition de l'objectif initial des concepteurs (il s'agit de la restructuration des éléments de l'objet connecté par les utilisateurs pour créer quelque chose de nouveau), la routinisation des usages extra rôles (il s'agit de la stabilisation des usages discrétionnaires dans la vie quotidienne en investissant des efforts supplémentaires, en passant plus de temps, au-delà de ce qui est initialement attendu par les fabricants, afin de contribuer davantage à l'objet connecté). À un faible niveau d'appropriation par l'utilisateur, les objets connectés sont utilisés tels qu'ils ont été initialement conçus. Les consommateurs répètent les usages maîtrisés sur leurs autres appareils mobiles et personnalisent leurs objets connectés comme prévus par les concepteurs. À un niveau élevé d'appropriation par l'utilisateur, les consommateurs participent activement et subjectivement à l'élaboration des objets connectés en leur donnant un sens et en les utilisant d'une manière qui dépasse les objectifs initiaux des concepteurs. Les objets connectés sont utilisés comme co-créés lorsque leurs capacités technologiques sophistiquées permettent aux utilisateurs de façonner activement leurs expériences créées. Les objets connectés sont utilisés comme créés par soi-même lorsque leurs capacités technologiques ne permettent pas aux utilisateurs de co-créer pleinement des expériences, mais qu'ils sont redessinés par les utilisateurs pour répondre à leurs autres besoins personnels.

\section{Conclusion}

Les résultats de la présente recherche mettent en évidence les différents types d'objets connectés selon l'expérience utilisateur médiatisée par ces produits technologiques innovants, ainsi que la nature d'appropriation en fonction des modes d'usages effectifs, et soulignent la nécessité de prendre en compte le rôle crucial d'utilisateurs dans les écosystèmes des objets connectés.

\subsection{Contributions de recherche}

Sur le plan théorique, notre recherche présente plusieurs contributions. Premièrement, à notre connaissance, cette étude fait partie des premières études consacrées à la compréhension plus en profondeur sur les objets connectés grand public en se focalisant sur les expériences médiatisées par ces produits technologiques innovants et sur le niveau d'appropriation des utilisateurs. Deuxièmement, cette étude a permis de mieux comprendre la nature 
d'appropriation au niveau individuel, en identifiant quatre modes d'usages effectifs qui sont considérés comme des résultats du processus d'appropriation d'une technologie par les individus, ce qui est relativement peu exploré dans les travaux existants à ce jour. Troisièmement, cette étude permet d'enrichir la littérature relative à la création de valeur de la part des consommateurs par le biais de la cocréation d'expériences médiatisées par les technologies.

D'un point de vue managérial, les résultats de la présente recherche montrent un grand intérêt pour les acteurs du marché des objets connectés à destination du grand public, notamment les concepteurs d'objets connectés et les managers des points de vente. Le succès des produits technologiques innovants de consommation au marché est largement conditionné par la manière dont les consommateurs perçoivent, utilisent et intègrent le nouvel objet technologique dans leurs pratiques quotidiennes (Orlikowski, 1992; Rogers, 1983). Ainsi, pour les fabricants des objets connectés, il ne suffit pas de se concentrer sur les nouveaux services médiatisés par les technologies sophistiquées, et de négliger le potentiel de ce produit innovant laisse aux consommateurs pour se l'approprier. II est indispensable de développer des objets connectés en prenant en considération l'appropriabillité de ces produits technologiques innovants en émergence par les utilisateurs potentiels. Par ailleurs, les usages effectifs d'un même objet connecté peuvent varier d'un individu à l'autre, cette différence en termes de modes d'usages effectifs nous a servi d'un critère important pour proposer une nouvelle taxonomie des objets connectés en prisant en compte le rôle central d'utilisateurs. Les principaux résultats de cette étude nous ont permis de formuler un certain nombre de recommandations aux acteurs du marché des objets connectés à destination du grand public, spécifiques à chaque catégorie d'objets connectés.

- La catégorie des " objets connectés tels que conçus » : il s'agit des objets connectés qui sont utilisés tels qu'ils ont été initialement conçus. Les expériences médiatisées par cette catégorie d'objets connectés peuvent être améliorées par les capacités technologiques plus ou moins sophistiquées de ces objets connectés, néanmoins, le potentiel de cette catégorie d'objets connectés qui laisse aux consommateurs (utilisateurs potentiels) pour se l'approprier est relativement faible. Les consommateurs répètent les usages précédemment maîtrisés sur leurs autres appareils mobiles et personnalisent leurs objets connectés comme initialement prévus par les concepteurs. Pour les professionnels, il est recommandé de laisser un certain espace de liberté permettant aux clients potentiels de disposer de plus de possibilités d'appropriation pour faciliter l'intégration de l'objet connecté dans leurs pratiques quotidiennes. II est recommandé de proposer aux clients potentiels des objets connectés qui ont ajouté de nouveaux services à des objets d'usage familier. Ces objets connectés qui ne leur imposent pas d'adopter de nouvelles routines favorisent un usage pérenne de la part des clients potentiels.

- La catégorie des "objets connectés tels que co-créés": il s'agit des objets connectés qui sont utilisés tels qu'ils ont été co-créés par les concepteurs et les utilisateurs. Les capacités fonctionnelles de cette catégorie des objets connectés fournissent une variété de solutions 
technologiques permettant aux utilisateurs de participer activement à la cocréation d'expériences. De plus, le potentiel de cette catégorie d'objets connectés qui laisse aux consommateurs pour se l'approprier est relativement élevé. Les expériences médiatisées par cette catégorie d'objets connectés sont de nature enrichie par leurs capacités fonctionnelles liées à la haute technologie, ainsi que de nature habilitée par leurs capacités fonctionnelles liées à la technologie sophistiquée. C'est la catégorie des objets connectés la plus intéressante pour les professionnels du marché des objets connectés à destination du grand public. Face aux clients potentiels actifs et partagés, il est recommandé de considérer cette catégorie des objets connectés comme des composants informatiques ouverts qui nécessitent des mises à jour réguliers pour s'optimiser les services proposés. En raison de l'ajout d'une connexion réseau, la nature des objets physiques quotidiens se change radicalement en devant des mini-ordinateurs à titre principal. Ce qui implique un bouleversement dans les stratégies du développement des produits. Dans cette période de rupture technologique très forte, il est recommandé, pour les fabricants, de bien travailler sur le cycle d'évolution très court de leurs produits, et d'élargir régulièrement les possibilités de leurs produits en proposant de nouveaux services qui favorisent la création de valeur par le biais de la participation pérenne et active des utilisateurs dans la cocréation d'expériences.

La catégorie des "objets connectés tels que créés par soi-même ": il s'agit des objets connectés qui sont utilisés tels qu'ils sont créés par les utilisateurs eux-mêmes. Le potentiel de cette catégorie d'objets connectés qui laisse aux consommateurs (utilisateurs potentiels) pour se l'approprier est relativement élevé. Toutefois, les expériences médiatisées par cette catégorie d'objets connectés sont de nature assistée par leurs capacités technologiques les moins sophistiquées. Les objets connectés les plus simples qui ne fournissent que des informations, sont inclus dans cette catégorie. Pour les managers des points de vente, il est recommandé d'opter des vidéos ou messages publicitaires illustrant le potentiel d'application de ces objets connectés dans les différents contextes d'usage, en laissant un certain espace de liberté aux clients potentiels de créer un usage personnel et pérenne dans leurs pratiques quotidiennes.

\section{2 limites et perspectives de recherche}

La présente recherche n'est exempte de limites. L'ensemble de limites identifiées nous ont permis de faire émerger des voies futures de recherche. Tout d'abord, il serait pertinent pour les recherches futures de valider empiriquement sur un échantillon de consommateurs la taxonomie des objets connectés proposées dans le cadre de cette recherche. Par ailleurs, les objets connectés à destination du grand public véhiculent une véritable crainte d'atteinte à la vie privée dans le cadre du règlement général sur la protection des données (RGPD). II serait ainsi souhaitable de poursuivre notre étude en étudiant les expériences négatives 
médiatisées par les technologies ainsi que les modes de désappropriation associés. De plus, dans le cadre de cette étude, nous ne nous intéressons qu'aux utilisateurs dont l'âge varie de 18 ans à 60 ans. Afin de généraliser la taxonomie proposée, II serait pertinent de s'intéresser aux personnes plus âgées. Bien que cette cible soit probablement des réfractaires aux produits technologiques innovants, ils font partie des personnes les plus curieuses de connaître les dispositifs intelligents de santé. Ainsi, il serait intéressant pour les recherches futures de vérifier si les résultats peuvent être généralisés aux utilisateurs seniors qui ont plus de 60 ans dans le secteur de santé.

\section{Bibliographie :}

Ardelet, C., Veg-Sala, N., Goudey, A., \& Haikel-Elsabeh, M. (2017). Entre crainte et désir pour les objets connectés: Comprendre l'ambivalence des consommateurs. Décisions Marketing, 86, 31-46.

Atzori, L., lera, A., \& Morabito, G. (2014). From" smart objects" to" social objects": The next evolutionary step of the internet of things. IEEE Communications Magazine, 52(1), 97-105.

Bar, Pisani, F., \& Weber, M. (2016). Mobile technology appropriation in a distant mirror: Baroquization, creolization, and cannibalism. New Media \& Society, 18(4), 617-636.

Benghozi, P. J., Bureau, S., \& Massit-Folléa, F. (2015). L'Internet des objets/The Internet of Things: Quels enjeux pour l'Europe?/What Challenges for Europe? (MSH).

Carroll, J. (2004). Completing Design in Use : Closing the Appropriation Cycle. ECIS Proceedings, 44.

Carroll, J., Howard, S., Peck, J., \& Murphy, J. (2003). From adoption to use: the process of appropriating a mobile phone. Australasian Journal of Information Systems, 10(2), 38-48.

Chouk, I., \& Mani, Z. (2016). Les objets connectés peuvent-ils susciter une résistance de la part des consommateurs? Une étude netnographique. Décisions Marketing, 84, 19-41.

Fortino, G., Rovella, A., Russo, W., \& Savaglio, C. (2014). On the Classification of Cyberphysical Smart Objects in the Internet of Things. In UBICITEC (pp. 8694).

Gavard-Perret, M., Gotteland, D., Haon, C., Aubert, B., Avenier, M., Duymedjian, R., A. M.-J. (2012). Méthodologie de la recherche en sciences de gestion réussir son mémoire ou sa thèse (2e édition). Pearson Education.

GFK. (2014). objets connectés: un tiers des utilisateurs les abandonnent dans les 6 
mois. https://www.phonandroid.com/objets-connectes-tiers-utilisateurs-lesabandonnent-6-mois.html\#ixzz3KfF9i3aA

Hernández, M. E. P., \& Reiff-Marganiec, S. (2014). Classifying smart objects using capabilities. International Conference on SMARTCOMP (IEEE), 309-316.

IoT Analytics. (2018). State of the IoT 2018: Number of loT devices now at $7 B-$ Market accelerating. https://iot-analytics.com/state-of-the-iot-update-q1-q22018-number-of-iot-devices-now-7b/

Kawsar, F., Nakajima, T., Park, J. H., \& Yeo, S. S. (2010). Design and implementation of a framework for building distributed smart object systems. The Journal of Supercomputing, 54(1), 4-28.

Kiritsis, D. (2011). Closed-loop PLM for intelligent products in the era of the Internet of things. Computer-Aided Design, 43(5), 479-501.

Kortuem, G., Kawsar, F., Sundramoorthy, V., \& Fitton, D. (2010). Smart Objects as Building Blocks for the Internet of Things. IEEE Internet Computing, 14(1), 44-51. https://doi.org/10.1109/MIC.2009.143

López, T. S., Ranasinghe, D. C., Patkai, B., \& McFarlane, D. (2011). Taxonomy, technology and applications of smart objects. Information Systems Frontiers, 13(2), 281-300.

McKinsey Global Institute. (2015). The internet of things: mapping the value beyond the hype. https://www.mckinsey.com/ /media/McKinsey/Business Functions/McKinsey Digital/Our Insights/The Internet of Things The value of digitizing the physical world/Unlocking_the_potential_of_the_Internet_of_Things_Executive_summa ry.ashx

Meyer, G. G., Främling, K., \& Holmström, J. (2009). Intelligent products: A survey. Computers in Industry, 60(3), 137-148.

Moawad, A., Efthymiou, V., Caire, P., Nain, G., \& Le Traon, Y. (2012). Introducing conviviality as a new paradigm for interactions among IT objects.

Proceedings of the Workshop on Al Problems and Approaches for Intelligent Environments, (Vol. 907, pp. 3-8). CEUR-WS. org.

Neuhofer, B., Buhalis, D., \& Ladkin, A. (2014). A typology of technology enhanced tourism experiences. International Journal of Tourism Research, 16(4), 340350.

Ning, H., \& Hu, S. (2012). Technology classification, industry, and education for Future Internet of Things. International Journal of Communication Systems, 25(9), 1230-1241.

Oberländer, A. M., Röglinger, M., Rosemann, M., \& Kees, A. (2018).

Conceptualizing business-to-thing interactions-A sociomaterial perspective on the Internet of Things. European Journal of Information Systems, 27(4), 486-502. 
Orlikowski, W. J. (1992). The duality of technology: rethinking the concept of technology in organizations. Organization Science, 3(3), 398-427. https://doi.org/10.2307/2635280

Orlikowski, W. J. (2000). Using Technology and Constituting Structures: A Practice Lens for Studying Technology in Organizations. Organization Science, 11(4), 404-428. https://doi.org/10.1287/orsc.11.4.404.14600

Porter, M. E., \& Heppelmann, J. E. (2014). How Smart, Connected Products Are Transforming Competition. Harvard Business Review, 92(11), 64-88. https://doi.org/10.1017/CBO9781107415324.004

Püschel, L., Röglinger, M., \& Schlott, H. (2016). What's in a Smart Thing? Development of a Multi-Layer Taxonomy. 37th International Conference on Information Systems (ICIS), 4801, 1-19.

Rogers E. M. (1983). Diffusion of innovations (Free Press).

Sandström, S., Edvardsson, B., Kristensson, P., \& Magnusson, P. (2008). Value in use through service experience. Managing Service Quality, 18(2), 112-126.

Smutný, P. (2016). Different perspectives on classification of the Internet of Things. Carpathian Control Conference (ICCC), 2016 17th International, IEEE, 692696.

Sundmaeker, H., Guillemin, P., Friess, P., \& Woelfflé, S. (2010). Vision and challenges for realising the Internet of Things. Cluster of European Research Projects on the Internet of Things, European Commision, 3(3), 34-36.

The Carnegie Mellon University Computer Science Department Coke Machine. (1990). The "Only" Coke Machine on the Internet. http://www.cs.cmu.edu/ coke/history_long.txt

Uckelmann, D., Harrison, M., \& Michahelles, F. (2011). An architectural approach towards the future internet of things. In Architecting the internet of things (pp. 1-24). Springer.

Yoo, Y., Henfridsson, O., \& Lyytinen, K. (2010). Research commentary-the new organizing logic of digital innovation: an agenda for information systems research. Information Systems Research, 21(4), 724-735.

Yoo, Y. (2010). Computing in everyday life: A call for research on experiential computing. MIS Quarterly, 213-231.

Zhong, Balagué \& Benamar, Z. (2017). Outcomes of Consumer loT Device Appropriation: A Study of consumers' daily effective uses of smart watches. Proceedings of the 33th International AFM (French Marketing Academy) Congress.

Zhong, Z. (2019). Comprendre l'appropriation des objets connectés grand public : une approche de modélisation à composants hiérarchiques. In Doctoral Dissertation. Université Paris Saclay. 


\section{Annexe 1 : Synthèse des caractéristiques fonctionnelles perçues d'objets connectés}

\begin{tabular}{|c|c|c|}
\hline Thème & Sous-thème & Exemple de verbatim \\
\hline Identification & $\begin{array}{l}\text { Objet } \\
\text { identifiable }\end{array}$ & $\begin{array}{l}\text { "Grâce à des tags RFID, ces objets } \\
\text { peuvent s'identifier, ce qui leur permet de } \\
\text { se connecter et de communiquer } \\
\text { efficacement avec d'autres objets " } \\
\text { [E16V4c] }\end{array}$ \\
\hline Communication & $\begin{array}{l}\text { Objet } \\
\text { communicant }\end{array}$ & $\begin{array}{l}\text { "C'est un objet qui communique avec } \\
\text { Internet, qui envoie des informations qui } \\
\text { peut en recevoir » [E6V1] } \\
\text { "Pour moi un objet connecté c'est un } \\
\text { objet qui va communiquer avec d'autres } \\
\text { objets" [E14V1a] }\end{array}$ \\
\hline Réseautage & $\begin{array}{l}\text { Interaction avec } \\
\text { des services } \\
\text { Echange des } \\
\text { données }\end{array}$ & $\begin{array}{l}\text { "Un objet qui permet, en mode SAAS } \\
\text { généralement, d'interagir avec d'autres } \\
\text { objets" [E1V1a] } \\
\text { "Un objet connecté c'est un objet heu } \\
\text { pour lequel je pourrais avoir des } \\
\text { informations ou envoyer des informations } \\
\text { à distance, par internet ou par autre } \\
\text { moyen" [E12V4]. } \\
\text { "Objet qui peut envoyer et recevoir des } \\
\text { données à distance via une connectivité } \\
\text { soit locale, soit générale. " [E9V1.1] }\end{array}$ \\
\hline $\begin{array}{l}\text { Détection \& } \\
\text { actionnement }\end{array}$ & $\begin{array}{l}\text { Capture des } \\
\text { données } \\
\text { Déclenchement }\end{array}$ & $\begin{array}{l}\text { « Et qui va aussi capter des données, qui } \\
\text { va m'envoyer, voilà » [E4V2b] } \\
\text { «J'ai également une prise à déclencheur }\end{array}$ \\
\hline
\end{tabular}




\begin{tabular}{|c|c|c|}
\hline & d'une action & $\begin{array}{l}\text { avec mon téléphone pour mettre le } \\
\text { chauffage en route par exemple, qui m'est } \\
\text { utile quand je voyage, quand j'arrive je } \\
\text { déclenche la chaudière " [E10V1e]. }\end{array}$ \\
\hline Autogestion & $\begin{array}{l}\text { Apprentissage à } \\
\text { partir des } \\
\text { informations } \\
\text { récoltées } \\
\text { Adaptation aux } \\
\text { situations }\end{array}$ & $\begin{array}{l}\text { "Avec des capacités d'apprentissage, } \\
\text { plus ces objets savent sur nous, plus ils } \\
\text { pourront réagir de manière intelligente } \\
\text { devant nous "[E15V18d] } \\
\text { "Ces objets savent s'adapter à nous, à } \\
\text { l'environnement "[E2V20c] }\end{array}$ \\
\hline $\begin{array}{l}\text { Traitement \& } \\
\text { programmation }\end{array}$ & $\begin{array}{l}\text { Capacité de } \\
\text { traitement } \\
\text { Programmation } \\
\text { des tâches }\end{array}$ & $\begin{array}{l}\text { "Tout objet quel que soit sa taille qui peut } \\
\text { être connecté, recevoir des informations } \\
\text { et les traiter » [E6V1d] } \\
\text { "Ces objets peuvent, en fonction de leur } \\
\text { programmation, alerter l'utilisateur ou } \\
\text { piloter des choses » [E14V16c] }\end{array}$ \\
\hline
\end{tabular}

Annexe 2: Exemples d'usages effectifs par modes identifiés dans le processus d'appropriation de la montre connectée

\begin{tabular}{|l|l|l|l|l|}
\hline & $\begin{array}{l}\text { Répétition } \\
\text { d'usages } \\
\text { antérieurs } \\
\text { maitrisés }\end{array}$ & Personnalisation & $\begin{array}{l}\text { Redéfinition } \\
\text { de l'objectif } \\
\text { initial des } \\
\text { concepteurs }\end{array}$ & $\begin{array}{l}\text { Routinisation } \\
\text { des usages } \\
\text { extra rôles }\end{array}$ \\
\hline $\begin{array}{l}\text { Interaction } \\
\text { sensorielle }\end{array}$ & $\begin{array}{l}\text { Regard et } \\
\text { Toucher des } \\
\text { différentes } \\
\text { parties de la } \\
\text { montre } \\
\text { (l'écran } \\
\text { tactile, } \\
\text { bracelet, } \\
\text { etc.) }\end{array}$ & & \\
& $\begin{array}{l}\text { Observation } \\
\text { des effets à } \\
\text { l'allumage, la } \\
\text { luminosité, } \\
\text { etc. }\end{array}$ & & \\
\hline $\begin{array}{l}\text { Interaction } \\
\text { de } \\
\text { manipulation }\end{array}$ & $\begin{array}{l}\text { Manipulation } \\
\text { de différents } \\
\text { boutons, de }\end{array}$ & & & \\
\hline
\end{tabular}




\begin{tabular}{|c|c|c|c|c|}
\hline & l'écran tactile & & & \\
\hline $\begin{array}{l}\text { Interaction } \\
\text { fonctionnelle }\end{array}$ & $\begin{array}{l}\text { Test de } \\
\text { fonctions } \\
\text { basiques en } \\
\text { général } \\
\text { Installation } \\
\text { d'application } \\
\text { s pour } \\
\text { augmenter et } \\
\text { diversifier les } \\
\text { usages }\end{array}$ & $\begin{array}{l}\text { Tri de } \\
\text { fonctionnalités } \\
\text { selon l'utilité } \\
\text { perçue } \\
\text { Optimisation } \\
\text { des } \\
\text { fonctionnalités } \\
\text { et du } \\
\text { fonctionnement }\end{array}$ & $\begin{array}{l}\text { Utilisation } \\
\text { experte et } \\
\text { créative des } \\
\text { fonctionnalité } \\
\text { s } \\
\text { par } \\
\text { exemple : } \\
\text { Alarme du } \\
\text { commencem } \\
\text { ent du dîner } \\
\text { (fonction } \\
\text { initialement } \\
\text { conçue : } \\
\text { recherche de } \\
\text { smartphone } \\
\text { avec la } \\
\text { montre } \\
\text { connectée en } \\
\text { faisant } \\
\text { sonner le } \\
\text { smartphone) }\end{array}$ & $\begin{array}{l}\text { Installation } \\
\text { régulière de } \\
\text { nouvelles } \\
\text { applications } \\
\text { pour } \\
\text { compléter } \\
\text { des } \\
\text { fonctionnalité } \\
\text { s } \\
\text { manquantes } \\
\text { de la montre } \\
\text { connectée } \\
\text { (par } \\
\text { exemple } \\
\text { Notifications } \\
\text { sur le } \\
\text { téléphone } \\
\text { lorsque la } \\
\text { batterie de la } \\
\text { montre } \\
\text { connectée } \\
\text { sera bientôt } \\
\text { épuisée) }\end{array}$ \\
\hline $\begin{array}{l}\text { Interaction } \\
\text { cognitive }\end{array}$ & & $\begin{array}{l}\text {-Recherche } \\
\text { ciblée de } \\
\text { nouvelles } \\
\text { applications } \\
\text { Test de } \\
\text { nouvelles } \\
\text { applications }\end{array}$ & $\begin{array}{l}\text { Interrogation } \\
\text { s sur le } \\
\text { fonctionneme } \\
\text { nt de la } \\
\text { montre } \\
\text { connectée, et } \\
\text { sur ses } \\
\text { fonctionnalité } \\
\text { s } \\
\text { Essai de } \\
\text { différentes } \\
\text { fonctionnalité } \\
\text { s (essai et } \\
\text { erreur) } \\
\text { Essai de } \\
\text { différentes } \\
\text { applications }\end{array}$ & \\
\hline $\begin{array}{l}\text { Interaction } \\
\text { expressive }\end{array}$ & & $\begin{array}{l}\text { Assortiment du } \\
\text { cadran avec la }\end{array}$ & $\begin{array}{l}\text { Personnalisa } \\
\text { tion du }\end{array}$ & $\begin{array}{l}\text { Personnalisa } \\
\text { tion régulière }\end{array}$ \\
\hline
\end{tabular}




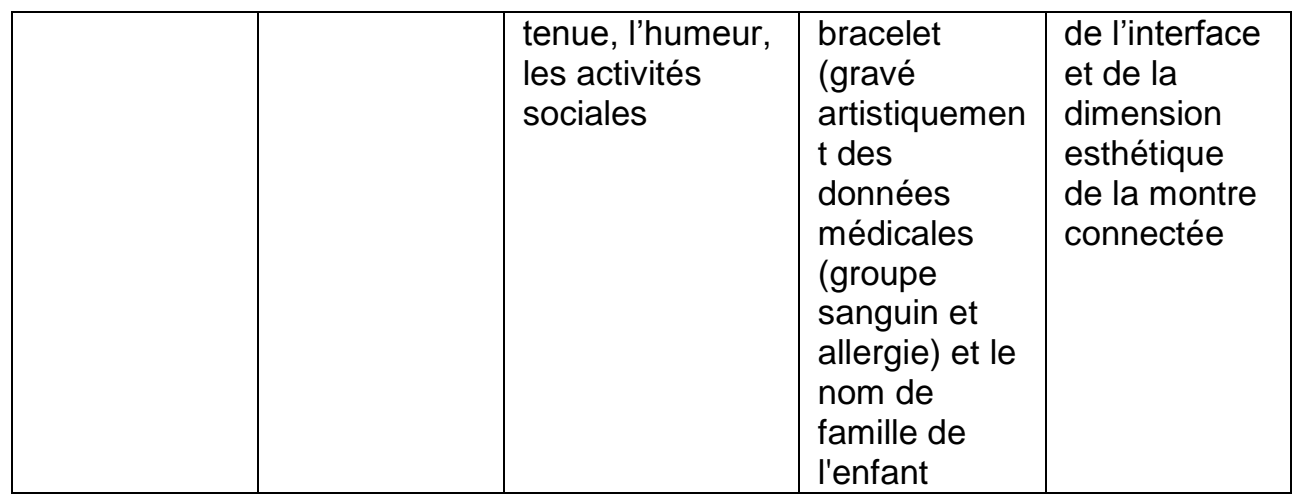

